\title{
Gender Differences in Substance Use Across Marital Statuses
}

\author{
Sampson Lee Blair ${ }^{1}$ and Melissa A. Menasco, ${ }^{2,}$ \\ ${ }^{1}$ Dept. of Sociology, The State University of New York, USA \\ ${ }^{2}$ Criminal Justice Department, Buffalo State, USA
}

\begin{abstract}
Previous studies have noted that the relationship status of adults is substantially linked with levels of substance use. Understandably, the marital status of adults continues well beyond its initial phases, sometimes resulting in divorce, separation, or remarriage. This study seeks to extend our understanding of the linkages between marital status and substance use among adults. Using data from the 2012 National Survey on Drug Use and Health, we examine the substance use levels among a nationally representative sample of 14,715 adults. The analyses indicate that, for both females and males, marriage is, indeed, associated with lower levels of alcohol, cigarette, and marijuana use. Divorced individuals reported the highest levels of substance use. Interestingly, remarried individuals report higher levels of substance use than their counterparts in their first marriage, yet remarried men and women report lower levels of usage than do those who are currently divorced. Contextual and individual characteristics also yield several interesting patterns. In particular, distress and depression are shown to be much stronger predictors of substance use levels among divorced and remarried individuals. Divorced and remarried women, as compared to their male counterparts, are shown to be significantly more influenced by their employment status. The implications of this study are discussed, as are the potentially reciprocal nature of marital status and substance use.
\end{abstract}

Keywords: Substance use, marriage, divorce, remarriage, gender.

Since World War II, the American population has experienced a considerable shift in many of its basic demographic traits, not the least of which pertain to the marital statuses of individuals. The post-war baby boom, with its tremendous surge in fertility rates, was followed by higher rates of divorce, which began to appear during the 1960s, peaking in the early 1980s. During the 1990s, marriage rates began to decline, the percentage of remarried partners rose, and cohabitation rates continued to climb (Kreider and Fields, 2002). Over the past decade, the percentage of individuals choosing to remain single continues to rise (U.S. Census Bureau, 2012). Understandably, these changes are not without consequences, particularly in regard to individuals' behavioral patterns.

Previous studies have demonstrated a linkage between marital status and substance use, yet the vast majority of such studies have focused upon the transition into marriage (e.g., Fleming et al., 2010; Leonard and Homish, 2005), and have not examined more fully the range of additional marital statuses. Researchers have noted that married individuals tend to consume less substances as compared to their single counterparts. This tendency, often referred to as the "marriage effect," has been associated with decreases in drinking, smoking, and marijuana usage among married persons (Bachman et al., 2002; Merline et al., 2008). Marital dissolution, on the other hand, has

*Address correspondence to this author at the Criminal Justice Department, Buffalo State, Buckham Hall A100E, 1300 Elmwood Avenue, Buffalo, NY 14222-1095, USA; Tel: 1-716-878-3228; Fax: 1-716-878-3240;

E-mail: menascma@buffalostate.edu been associated with increases across the same substances (Merline et al., 2008). This study attempts to examine one of the more serious issues, substance use, as it varies across the marital statuses of individuals, as well as how it varies in regard to gender.

\section{THE NATURE OF MARITAL STATUS}

Although marital forms have become increasingly varied over recent decades, marriage itself is still largely considered to be advantageous to individuals. On average, married individuals tend to have significantly higher household incomes (U.S. Census Bureau, 2012), and enjoy a higher standard of living, as compared to single persons. In terms of physical health and mental well-being, married individuals tend to fare much better than their single counterparts, on average (Horowitz et al., 1996). Even demographic shifts, such as the increase in cohabitation rates and singlehood, have not taken away from individuals' desire to marry. Research has shown that the overwhelming majority of young adults still regard marriage as an important status goal to be achieved (Blair, 2010). For many individuals, particularly those in late adolescence and early adulthood, the establishment of long-term intimate relationships is regarded as a central and even essential element of becoming an adult (Graber and Brooks-Gunn, 1996).

Divorce has been widely examined by family researchers over the past half of a century, with most studies pointing towards the wide variety of potential effects which it can have upon both adults and children. 
One of the leading complications of divorce is, understandably, the economic consequences which follow, as many studies have demonstrated the substantially more harmful financial impact of divorce upon women, who are much more likely to fall into poverty after a divorce, as compared to men (U. S. Census Bureau, 2012). Among males, divorce has even been shown to improve their economic well-being (Sweeney, 1997).

The unique qualities of remarriage, in comparison to first marriage, tend to make remarried relationships considerably more complicated, and perhaps even induce instability. Remarried individuals often enter into a new union, while still maintaining both parental roles and relationships with their former spouses, thereby making it necessary to formulate new relationships with their current spouse, maintain relationships with their children from a previous marriage, establish ties with their new relatives (the kin of their new spouse), and attempt to balance this network of relationships appropriately (Fischer et al., 2005). Remarriages are often fraught with higher levels of stress, and relationship quality between individuals and their new spouses, as well as with both previous children and new children, often suffers (Ehrenberg, et al., 2012; Mirecki et al., 2013). The somewhat fragile nature of remarriage can also be seen in its dissolution rate, which is considerably higher than that of first marriages (Saint-Jacques et al., 2011). Of course, remarriage occurs at a later stage in life for individuals, so their ability and willingness to adapt to new relationships may be confounded by earlier experiences (Sweeney, 1997).

\section{SUBSTANCE USE, MARITAL STATUSES, AND GENDER}

The very nature of marriage is one which often is associated with maturation and the adoption of more adult-like responsibilities. When the individual makes the transition from single to married, there is inherently a movement away from a single lifestyle, often focused on individual desires and goals, to a partnered lifestyle which is centered on common goals and the maintenance of long-term, mutual satisfaction (McGoldrick and Carter, 1982). Marital responsibilities can range from the daily, such as parenting and spousal relations, to the more sporadic, such as maintaining ties with extended kin, yet the overall tendency, once married, is for individuals to accept the variety of roles and attempt to accommodate them by changing their own lifestyles. Researchers have often regarded marriage as having a suppressive effect upon a variety of deleterious issues, ranging from morbidity, mental well-being, psychological distress, to even crime and violence (Horney et al., 1995; Horwitz et al., 1996), and particularly so for men. Such benefits of marriage have been associated with the greater availability of support networks which married life typically brings about, as well as the element of social control inherent within marriage (Maume et al., 2005).

In regard to substance use, researchers have noted that, in the transition from late adolescence into adulthood, individuals do tend to use increasingly less (Leonard and Homish, 2005). The consumption of alcohol and marijuana, for instance, have been shown to steadily decline as individuals approach their mid20 s, which is also when many are entering into either marriage or relatively stable relationships, such as cohabitation (Chen and Kandel, 1995; Muthen and Muthen, 2000). Indeed, it has been posited that substance use levels are strongly linked to the variety of responsibilities which individuals bear (Bachman et al., 2002). If so, marriage and family roles would certainly weigh heavily upon an individual's choices concerning substance use, and would tend to be associated with lower rates of usage. Even the surrounding social context of individuals can be affected by marital status, as it has been proposed that marriage itself can lead to less interaction with others who use substances (Bachman et al., 2002). Hence, from a functionalist perspective, the responsibilities and shared goals of marital relationships serve to make individuals adhere to more familistic orientations, while having less individualistic orientations. A lower level of substance use would allow individuals to conform more readily to those more familistic expectations. Overall, being married may be linked with both more responsible values and a distinct support network, and the combined influences of these are more compatible with lower rates of substance use (i.e., more responsible behavior)(Giordarno et al., 2002). Being single, by either non-marriage or divorce, may lead to the opposite set of qualities.

Most studies which have directly compared the substance use rates of women and men have noted that women tend to use substantially lower amounts of almost all substances, as compared to men (Merline et al., 2008). These differences in usage patterns are particularly evident in regard to heavy usage rates, wherein women are sharply less likely to drink, smoke, or use illicit drugs (Substance Abuse and Mental Health Services Administration, 2003). It is difficult, if not 
impossible, to disentangle the influences of marital status and gender. Although American couples have steadily become more egalitarian over the past half of a century, marital and familial roles are still rather segregated. However, research has shown that the birth of a child tends to prompt fathers to spend less time with their male peers, less time frequenting bars and other sorts of social activities involving peers and alcohol, and to spend greater amounts of time engaged with their spouse and children (Bachman et al., 2002). If these patterns are due to prevailing social norms concerning men's roles within a specific marital or parental context are true, then it logically follows that men who are not married or who are not fathers do not feel the same social constraints, nor do they necessarily feel compelled to lower their rates of substance use.

Societal pressures and prevailing norms concerning marital and familial roles are not without a strong, gendered quality. In the case of males, the desire to be the breadwinner of the family and to become a father may often be regarded as an expression of masculinity (e.g., Nock, 1998). Women are not immune to these same social constraints, as research has clearly shown that women, when expecting a baby, will either sharply curtail their rates of drinking, smoking, and the use of other illicit substances, or they will cease using them, altogether (Bachman et al., 1997). For both sexes, the adoption of and adherence to societal norms concerning various marital and familial roles may still have a quite salient and substantial bearing upon their choices concerning substance use.

\section{DATA}

Data for this study are taken from the 2012 wave of the National Survey on Drug Use and Health (NSDUH). The sample used herein is a nationally representative sample of adults in the United States, 18 years of age and older. Initiated in 1971, this cross-sectional survey attempts to gauge a wide variety of substance use behaviors, both current and past. Respondents in this study are assured of the confidentiality of their answers; hence, the data used in this study are regarded as both reliable and accurate. After removing cases due to missing data, the resulting sample is 19,382 females and 18,332 males. Given the nature of these analyses, the sample was separated into four marital status groups: those who were in their first marriage, those who were in a second or subsequent marriage, those who were currently divorced, and those who were never married.
Several measures are used in order to assess the substance use patterns of respondents. Although there are certainly a wide variety of both substances and particular forms of use among adults, this study will concentrate on three specific types - alcohol, cigarettes, and marijuana. The availability of and access to these substances are obviously quite disparate, in addition to their effects upon the human body using these three should provide a range of substance use which is representative of adult usage patterns in the United States. For each substance, respondents were queried as to how many times, over the past month, they had used each substance.

A variety of individual and household characteristics were included in the analyses. Educational attainment was measured as a four-point scale, ranging from (1) less than a high school degree, (2) a high school degree, (3) some college, to (4) a college degree. The age of respondents was recoded as an eleven-point scale, which ranged as follows: $7=18$ years of age, $8=19$ years of age, $9=20$ years of age, $10=21$ years of age, $11=22$ or 23 years of age, $12=24$ or 25 years of age, $13=26$ to 29 years of age, $14=30$ to 34 years of age, $15=35$ to 49 years of age, $16=50$ to 64 years of age, and $17=65$ years of age or older. Annual household income was assessed on a seven-point scale, ranging from (1) less than $\$ 10,000$, (2) $\$ 10,000$ to $\$ 19,999$, (3) $\$ 20,000$ to $\$ 29,999$, (4) $\$ 30,000$ to $\$ 39,999$, (5) $\$ 40,000$ to $\$ 49,999$, (6) $\$ 50,000$ to $\$ 74,999$, and (7) $\$ 75,000$ or more. In addition, respondents were queried about their use of either supplemental security income (SSI), food stamps, or either cash or noncash government assistance (coded as $1=y e s$ to any of the forms of assistance, $0=$ none).

Given that the presence of children may influence the propensity to use various substances, the number of children in the home was included in the analyses. Respondents were also asked whether they were currently employed, at least part-time (20 or more hours each week), with responses coded as $1=$ currently employed, $0=$ not currently employed. As discussed previously, stressors such as moving have been shown to be associated with substance use levels. Hence, respondents were asked how many times they had moved within the previous five years (coded as $0=$ never to $5=$ five or more times). Respondents were also asked how frequently they had attended religious services over the previous twelve months. Responses to this question ranged from: (1) never, (2) once or twice, (3) three to five times, (4) six 
to twenty-four times, (5) twenty-five to fifty-two times, and (6) more than fifty-two times.

The linkages between well-being and substance use have been clearly substantiated in previous studies. As such, several measures of well-being are included in these analyses. The physical health of respondents was obtained with the following question: "Would you say that your health, in general, is excellent (1), very good (2), good (3), fair (4), or poor (5)?" Psychological distress was measured using a nonspecific psychological distress scale (Furukawa, Kessler, Slade, \& Andrews, 2003; Kessler et al., 2003). This scale was created from responses to six different questions. These included: "During the past 30 days, how often did you feel nervous?," "During the past 30 days, how often did you feel hopeless?," "During the past 30 days, how often did you feel restless or fidgety?," "During the past 30 days, how often did you feel so sad or depressed that nothing could cheer you up?," "During the past 30 days, how often did you feel that everything was an effort?," and "During the past 30 days, how often did you feel down on yourself, no good, or worthless?" The responses to each of these questions ranged from (1) all of the time to (5) none of the time. The six questions were used to create a single index measure of psychological distress (Cronbach's alpha $=0.83$ ). A measure of depression was also utilized, with respondents answering the question: "Have you ever in your life had a period of time lasting several days or longer when most of the day you felt sad, empty or depressed?" Responses to this item were coded as (1) yes or (0) no.

\section{RESULTS}

Table 1 presents the mean levels of substance use, by marital status, for females and males. In addition, the overall rates of usage are presented for each respective category of substance and marital status. Among individuals in their first marriage, males report significantly higher levels of usage across alcohol, cigarettes, and marijuana, as compared to females. The disparity between female and male usage rates is particularly noteworthy in regard to marijuana, where first-married males have a usage rate which is approximately three times higher than that of firstmarried females.

Among remarried individuals, males are again shown to report significantly higher levels of alcohol and marijuana usage, as compared to females (6.32 versus 4.22 and 0.91 versus 0.51 , respectively).
However, remarried females report a slightly higher rate of cigarette use, as compared to males (although the difference is not statistically significant). As compared to their first-married counterparts, remarried individuals appear to have higher levels of alcohol and cigarette usage, but report comparable levels of marijuana use.

Divorced individuals seem to have a rather unique pattern of substance use, as compared to individuals of other marital statuses. Divorced males, once again, report significantly higher levels of alcohol, cigarette, and marijuana usage, as compared to divorced females. When compared to first-married and remarried individuals, both divorced females and males seem to smoke considerably more cigarettes than do individuals of other marital statuses. As alluded to earlier, this may result from the greater stress associated with divorce (e.g., as a coping mechanism) or the smoking itself may have contributed to the dissolution of the marital relationship. These possibilities will be discussed at length in due course. It is also interesting to note that divorced females appear to drink alcohol less frequently, as compared to remarried females, while the opposite is shown among divorced males and remarried males.

Among never-married individuals, males have significantly higher rates of usage across all three of the measured substances. Given that previous research has consistently shown males to have higher rates of substances, as compared to females, this finding is to be expected. It is worth noting, though, that both never-married females and males report substantially higher rates of marijuana use, as compared to individuals from all other marital statuses. Understandably, a large portion of the never-married population is in their early adult years, which are typically more likely to use marijuana. Indeed, approximately $11.2 \%$ of never-married males and $5.3 \%$ of never-married females report using marijuana 20 or more times over the previous month, strongly suggesting a habitual pattern of usage.

Table 2 presents the mean levels of household and individual characteristics, by sex and marital status. In regard to educational attainment, females across all marital statuses reported significantly higher levels of educational attainment, as compared to males. This is consistent with current population trends in the United States, such that more females are attaining college degrees, as compared to males. With the exception of never-married individuals, males were consistently 
Table 1: Mean levels of Substance Use and Age at First Use, by Marital Status

\begin{tabular}{|c|c|c|c|c|c|c|c|c|}
\hline & \multicolumn{2}{|c|}{ First Marriage } & \multicolumn{2}{|c|}{ Remarriage } & \multicolumn{2}{|c|}{ Divorced } & \multicolumn{2}{|c|}{ Never Married } \\
\hline & Females & Males & Females & Males & Females & Males & Females & Males \\
\hline \multicolumn{9}{|c|}{ Use Past 30 Days } \\
\hline Alcohol & $\begin{array}{r}3.24 \\
(5.90)\end{array}$ & $\begin{array}{c}5.45^{\star \star \star} \\
(7.75)\end{array}$ & $\begin{array}{c}4.22 \\
(7.24)\end{array}$ & $\begin{array}{l}6.32^{\star \star *} \\
(8.95)\end{array}$ & $\begin{array}{c}3.87 \\
(6.51)\end{array}$ & $\begin{array}{c}6.76^{\star \star *} \\
(8.93)\end{array}$ & $\begin{array}{c}3.74 \\
(5.57)\end{array}$ & $\begin{array}{c}5.19^{* * *} \\
(7.01)\end{array}$ \\
\hline Cigarettes & $\begin{array}{r}3.77 \\
(9.60)\end{array}$ & $\begin{array}{l}4.77^{* \star *} \\
(10.50)\end{array}$ & $\begin{array}{c}6.96 \\
(12.29)\end{array}$ & $\begin{array}{c}6.86 \\
(12.23) \\
\end{array}$ & $\begin{array}{c}9.26 \\
(13.36)\end{array}$ & $\begin{array}{l}11.07^{\star * *} \\
(13.81)\end{array}$ & $\begin{array}{c}6.13 \\
(11.35)\end{array}$ & $\begin{array}{l}7.91^{* * *} \\
(12.22)\end{array}$ \\
\hline Marijuana & $\begin{array}{r}0.31 \\
(2.61)\end{array}$ & $\begin{array}{c}1.00^{* * *} \\
(4.83)\end{array}$ & $\begin{array}{c}0.51 \\
(3.44)\end{array}$ & $\begin{array}{l}0.91^{* *} \\
(4.56)\end{array}$ & $\begin{array}{c}0.84 \\
(4.23)\end{array}$ & $\begin{array}{c}1.88^{* * *} \\
(6.35)\end{array}$ & $\begin{array}{c}1.91 \\
(6.27)\end{array}$ & $\begin{array}{c}3.80^{\star * *} \\
(8.78)\end{array}$ \\
\hline \multicolumn{9}{|c|}{ Rate of Usage } \\
\hline \multicolumn{9}{|l|}{ Alcohol } \\
\hline Never & $49.5 \%$ & $37.1 \%$ & $44.9 \%$ & $40.3 \%$ & $44.5 \%$ & $37.7 \%$ & $41.1 \%$ & $36.6 \%$ \\
\hline 1-5 times & 33.4 & 33.1 & 34.2 & 27.7 & 35.4 & 26.4 & 36.7 & 32.4 \\
\hline $6-19$ times & 12.4 & 19.7 & 12.6 & 17.7 & 14.0 & 20.5 & 18.4 & 23.5 \\
\hline $20+$ times & 4.7 & 10.1 & 8.4 & 14.3 & 6.1 & 15.4 & 3.7 & 7.5 \\
\hline \multicolumn{9}{|l|}{ Cigarettes } \\
\hline Never & $83.8 \%$ & $78.8 \%$ & $73.0 \%$ & $72.9 \%$ & $62.3 \%$ & $54.8 \%$ & $70.2 \%$ & $60.8 \%$ \\
\hline 1-5 times & 2.7 & 3.7 & 2.3 & 2.7 & 4.8 & 5.1 & 6.7 & 8.7 \\
\hline $6-19$ times & 1.4 & 2.4 & 2.1 & 2.1 & 2.8 & 4.5 & 4.0 & 6.2 \\
\hline $20+$ times & 12.1 & 15.1 & 22.6 & 22.2 & 30.1 & 35.5 & 19.2 & 24.3 \\
\hline \multicolumn{9}{|l|}{ Marijuana } \\
\hline Never & $97.4 \%$ & $94.0 \%$ & $96.5 \%$ & $94.1 \%$ & $93.3 \%$ & $87.1 \%$ & $85.6 \%$ & $76.7 \%$ \\
\hline 1-5 times & 1.2 & 1.7 & 1.5 & 1.9 & 2.9 & 4.8 & 5.8 & 6.8 \\
\hline $6-19$ times & 0.5 & 1.3 & 0.5 & 1.2 & 1.5 & 2.7 & 3.2 & 5.3 \\
\hline $20+$ times & 0.9 & 3.0 & 1.5 & 2.7 & 2.2 & 5.4 & 5.3 & 11.2 \\
\hline $\mathrm{N}$ & 5886 & 4750 & 1329 & 1130 & 2209 & 1387 & 9958 & 10243 \\
\hline
\end{tabular}

Note: Significance levels indicate difference between the means of females and males within each marital status; Significance levels: ${ }^{* * *} p<.01,{ }^{* *} p<.05,{ }^{*} p<.10$

older than their female counterparts. Given the tendency of the marriage gradient among American couples, this is somewhat expected. Understandably, remarried individuals, both females and males, are substantially older than females and males of other marital statuses.

As compared head-to-head, it appears that firstmarried and remarried individuals report substantially higher levels of household income, as compared to both divorced and never-married individuals. Divorced females, more so than all others, report the lowest levels of household income. Not surprisingly, divorced females also reported the greatest use of welfare services (44\%), while first-married and remarried individuals reported the least. First-married individuals reported having the greatest numbers of children in the home (approximately 2.1 among first-married individuals), while never-married individuals reported the lowest. Across all marital status groups, males are shown to be significantly more likely to be employed outside the home, as compared to females. As anticipated, the difference between female and male employment rates is shown to be greatest among firstmarried individuals, where $81 \%$ of males were currently employed, as compared to $64 \%$ of females. Remarried individuals appear to be the least likely to have moved, while never-married individuals clearly are more geographically mobile, as compared to those of other marital statuses. In regard to religiosity, it appears that first-married individuals attend religious services more frequently than do individuals of other marital statuses, with divorced males and never-married females reporting the lowest levels of religious service attendance. 
Table 2: Mean Levels of Household and Individual Characteristics, by Marital Status

\begin{tabular}{|c|c|c|c|c|c|c|c|c|}
\hline & \multicolumn{2}{|c|}{ First Marriage } & \multicolumn{2}{|c|}{ Remarriage } & \multicolumn{2}{|c|}{ Divorced } & \multicolumn{2}{|c|}{ Never Married } \\
\hline & Females & Males & Females & Males & Females & Males & Females & Males \\
\hline Education & $\begin{array}{r}2.84 \\
(1.01)\end{array}$ & $\begin{array}{c}2.78^{* * *} \\
(1.07)\end{array}$ & $\begin{array}{c}2.69 \\
(0.94)\end{array}$ & $\begin{array}{c}2.55^{\star \star *} \\
(1.02)\end{array}$ & $\begin{array}{c}2.58 \\
(0.98)\end{array}$ & $\begin{array}{l}2.41^{\star * *} \\
(0.99)\end{array}$ & $\begin{array}{c}2.56 \\
(0.95)\end{array}$ & $\begin{array}{c}2.40^{\star * *} \\
(0.95)\end{array}$ \\
\hline Age & $\begin{array}{l}14.04 \\
(1.98)\end{array}$ & $\begin{array}{c}14.51^{* * *} \\
(1.76)\end{array}$ & $\begin{array}{l}15.29 \\
(1.18)\end{array}$ & $\begin{array}{c}15.62^{* * *} \\
(1.01)\end{array}$ & $\begin{array}{l}14.87 \\
(1.53)\end{array}$ & $\begin{array}{c}15.06^{* * *} \\
(1.34)\end{array}$ & $\begin{array}{l}10.68 \\
(2.41)\end{array}$ & $\begin{array}{l}10.72 \\
(2.42)\end{array}$ \\
\hline $\mathrm{HH}$ income & $\begin{array}{r}5.17 \\
(1.86)\end{array}$ & $\begin{array}{c}5.36^{* * *} \\
(1.77)\end{array}$ & $\begin{array}{c}5.30 \\
(1.78)\end{array}$ & $\begin{array}{c}5.36 \\
(1.75)\end{array}$ & $\begin{array}{c}3.71 \\
(2.00)\end{array}$ & $\begin{array}{l}4.03^{* * *} \\
(1.97)\end{array}$ & $\begin{array}{c}3.73 \\
(2.15)\end{array}$ & $\begin{array}{l}4.10^{* * *} \\
(2.15)\end{array}$ \\
\hline Welfare & $\begin{array}{r}0.18 \\
(0.38)\end{array}$ & $\begin{array}{c}0.16^{* * *} \\
(0.36)\end{array}$ & $\begin{array}{c}0.19 \\
(0.39)\end{array}$ & $\begin{array}{c}0.17 \\
(0.37)\end{array}$ & $\begin{array}{c}0.44 \\
(0.49)\end{array}$ & $\begin{array}{l}0.27^{\star * *} \\
(0.44)\end{array}$ & $\begin{array}{c}0.34 \\
(0.47)\end{array}$ & $\begin{array}{c}0.25^{\star * *} \\
(0.43)\end{array}$ \\
\hline \# of children & $\begin{array}{r}2.15 \\
(1.09)\end{array}$ & $\begin{array}{c}2.09^{\star * *} \\
(1.09)\end{array}$ & $\begin{array}{c}1.84 \\
(1.07)\end{array}$ & $\begin{array}{l}1.77^{*} \\
(1.04)\end{array}$ & $\begin{array}{c}1.86 \\
(1.04)\end{array}$ & $\begin{array}{l}1.50^{\star * *} \\
(0.88)\end{array}$ & $\begin{array}{c}1.71 \\
(0.95)\end{array}$ & $\begin{array}{c}1.48^{* * *} \\
(0.82)\end{array}$ \\
\hline Employment & $\begin{array}{r}0.64 \\
(0.48)\end{array}$ & $\begin{array}{c}0.81^{* * *} \\
(0.39)\end{array}$ & $\begin{array}{c}0.62 \\
(0.48)\end{array}$ & $\begin{array}{l}0.69^{\star * *} \\
(0.46)\end{array}$ & $\begin{array}{c}0.62 \\
(0.48)\end{array}$ & $\begin{array}{l}0.67^{\star * *} \\
(0.47)\end{array}$ & $\begin{array}{c}0.65 \\
(0.47)\end{array}$ & $\begin{array}{c}0.68^{\star * *} \\
(0.46)\end{array}$ \\
\hline $\begin{array}{l}\text { Recently } \\
\text { moved }\end{array}$ & $\begin{array}{r}1.18 \\
(1.52)\end{array}$ & $\begin{array}{l}1.00^{* * *} \\
(1.40)\end{array}$ & $\begin{array}{c}0.82 \\
(1.26)\end{array}$ & $\begin{array}{l}0.73^{*} \\
(1.11)\end{array}$ & $\begin{array}{c}1.41 \\
(1.63)\end{array}$ & $\begin{array}{r}1.42 \\
(1.59)\end{array}$ & $\begin{array}{c}1.77 \\
(1.74)\end{array}$ & $\begin{array}{l}1.57^{\text {** }} \\
(1.68)\end{array}$ \\
\hline Religiosity & $\begin{array}{r}3.29 \\
(1.93)\end{array}$ & $\begin{array}{c}3.03^{* * *} \\
(1.90)\end{array}$ & $\begin{array}{c}3.04 \\
(1.88)\end{array}$ & $\begin{array}{c}2.77^{\star * *} \\
(1.88)\end{array}$ & $\begin{array}{c}2.76 \\
(1.80)\end{array}$ & $\begin{array}{l}2.28^{* * *} \\
(1.64)\end{array}$ & $\begin{array}{c}2.65 \\
(1.75)\end{array}$ & $\begin{array}{c}2.33^{* * *} \\
(1.67)\end{array}$ \\
\hline $\begin{array}{l}\text { Physical } \\
\text { Health }\end{array}$ & $\begin{array}{r}2.16 \\
(0.93)\end{array}$ & $\begin{array}{c}2.22^{* * *} \\
(0.95)\end{array}$ & $\begin{array}{l}2.43 \\
(1.03)\end{array}$ & $\begin{array}{c}2.56^{\star * *} \\
(1.04)\end{array}$ & $\begin{array}{c}2.61 \\
(1.08)\end{array}$ & $\begin{array}{c}2.57 \\
(1.08)\end{array}$ & $\begin{array}{c}2.17 \\
(0.92)\end{array}$ & $\begin{array}{c}2.13^{\star * *} \\
(0.91)\end{array}$ \\
\hline Distress & $\begin{array}{r}3.72 \\
(4.01)\end{array}$ & $\begin{array}{l}3.13^{\star \star \star} \\
(3.62)\end{array}$ & $\begin{array}{l}3.98 \\
(4.50)\end{array}$ & $\begin{array}{l}3.14^{\star \star \star} \\
(3.89)\end{array}$ & $\begin{array}{c}5.26 \\
(5.26)\end{array}$ & $\begin{array}{l}4.28^{\star \star \star} \\
(4.68)\end{array}$ & $\begin{array}{l}5.52 \\
(4.83)\end{array}$ & $\begin{array}{l}4.80^{\star \star \star} \\
(4.70)\end{array}$ \\
\hline Depression & $\begin{array}{r}0.31 \\
(0.46)\end{array}$ & $\begin{array}{l}0.21^{* * *} \\
(0.40)\end{array}$ & $\begin{array}{c}0.41 \\
(0.49)\end{array}$ & $\begin{array}{l}0.25^{\star * *} \\
(0.43)\end{array}$ & $\begin{array}{c}0.45 \\
(0.49)\end{array}$ & $\begin{array}{l}0.34^{* * *} \\
(0.47)\end{array}$ & $\begin{array}{c}0.39 \\
(0.48)\end{array}$ & $\begin{array}{c}0.29^{\star * \star} \\
(0.45)\end{array}$ \\
\hline Black & $\begin{array}{r}0.07 \\
(0.25)\end{array}$ & $\begin{array}{c}0.07 \\
(0.24)\end{array}$ & $\begin{array}{c}0.07 \\
(0.25)\end{array}$ & $\begin{array}{l}0.09^{*} \\
(0.29)\end{array}$ & $\begin{array}{c}0.14 \\
(0.35)\end{array}$ & $\begin{array}{c}0.13 \\
(0.34)\end{array}$ & $\begin{array}{c}0.18 \\
(0.38)\end{array}$ & $\begin{array}{c}0.15^{\star * *} \\
(0.35)\end{array}$ \\
\hline Latino & $\begin{array}{r}0.16 \\
(0.36)\end{array}$ & $\begin{array}{c}0.16 \\
(0.36)\end{array}$ & $\begin{array}{c}0.10 \\
(0.30)\end{array}$ & $\begin{array}{c}0.10 \\
(0.30)\end{array}$ & $\begin{array}{c}0.14 \\
(0.35)\end{array}$ & $\begin{array}{c}0.13 \\
(0.33)\end{array}$ & $\begin{array}{c}0.17 \\
(0.37)\end{array}$ & $\begin{array}{l}0.18 \\
(0.38)\end{array}$ \\
\hline $\mathrm{N}$ & 5886 & 4750 & 1329 & 1130 & 2209 & 1387 & 9958 & 10243 \\
\hline
\end{tabular}

Note: Significance levels indicate difference between the means of females and males within each marital status; Significance levels: ${ }^{* * *} p<.01,{ }^{* *} p<.05,{ }^{*} p<.10$.

In terms of physical well-being, first-married males and remarried males reported significantly higher levels of physical health, as compared to their respective female counterparts. Across the various marital statuses, it appears that divorced males and females have the lowest levels of physical well-being. In regard to psychological distress, females in all four marital statuses report significantly higher levels of distress, as compared to males. Interestingly, divorced females and never-married females report the highest levels of psychological distress, while first-married males and remarried males report the lowest. Finally, in terms of depression, females are shown to report the highest levels, as compared to their male counterparts.
Divorced and remarried females report the highest levels of depression, while first-married and remarried males report the lowest.

Table 3 presents the results of ordinary least squares regression models of alcohol usage, as shown by sex and marital status. The models were all significant, and yielded between $5 \%$ to $10 \%$ of explained variance within each. Among first-married individuals, religiosity appears to provide a strong association with drinking, such that higher frequencies of religious service attendance are significantly associated with lower rates of consumption, for both females and males (beta $=-.11$ among females and - 
Table 3: Ordinary Least Squares Regression Models of Alcohol Usage, by Marital Status

\begin{tabular}{|c|c|c|c|c|c|c|c|c|}
\hline & \multicolumn{2}{|c|}{ First Marriage } & \multicolumn{2}{|c|}{ Remarriage } & \multicolumn{2}{|c|}{ Divorced } & \multicolumn{2}{|c|}{ Never Married } \\
\hline & Females & Males & Females & Males & Females & Males & Females & Males \\
\hline \multirow[t]{2}{*}{ Education } & $.09^{* * *}$ & $.04^{* *}$ & $.10^{\star * *}$ & $.09^{* \star \star}$ & $.08^{* * *}$ & .05 & $.12^{* \star *}$ & $.10^{* * *}$ \\
\hline & $(.25)$ & $(.16)$ & $(.41)$ & $(.40)$ & $(.26)$ & (.21) & $(.40)$ & $(.39)$ \\
\hline \multirow[t]{2}{*}{ Age } & $.11^{* \star *}$ & $.05^{\star * *}$ & $.07^{*}$ & -.01 & .02 & .01 & $.09^{* * *}$ & $.12^{\star \star \star}$ \\
\hline & $(.33)$ & $(.21)$ & $(.40)$ & $(-.08)$ & $(.07)$ & $(.05)$ & $(.21)$ & (.34) \\
\hline \multirow[t]{2}{*}{$\mathrm{HH}$ income } & $.10^{\star \star \star}$ & $.09^{* \star *}$ & .01 & $.07^{* *}$ & $.06^{\star *}$ & $.11^{* * *}$ & $.02^{\star *}$ & .01 \\
\hline & $(.32)$ & $(.41)$ & (.03) & $(.38)$ & (.18) & $(.49)$ & $(.06)$ & $(.02)$ \\
\hline \multirow[t]{2}{*}{ Welfare } & .01 & .01 & -.04 & .04 & $-.07^{\star \star *}$ & .04 & $-.05^{\star * *}$ & $-.05^{\star \star \star}$ \\
\hline & (.18) & $(.10)$ & $(-.65)$ & $(1.05)$ & $(-.96)$ & (.72) & $(-.62)$ & $(-.75)$ \\
\hline \multirow[t]{2}{*}{ \# of children } & $-.07^{\star \star *}$ & $-.03^{* *}$ & $-.07^{\star \star}$ & -.02 & -.01 & -.04 & $-.07^{\star \star *}$ & $-.05^{\star \star *}$ \\
\hline & $(-.35)$ & $(-.23)$ & $(-.45)$ & $(-.14)$ & $(-.04)$ & $(-.35)$ & $(-.41)$ & $(-.40)$ \\
\hline \multirow[t]{2}{*}{ Employment } & $.04^{* * *}$ & .02 & .01 & -.03 & .03 & $.07^{\star *}$ & $.06^{\star * *}$ & $.06^{\star \star *}$ \\
\hline & $(.45)$ & $(.31)$ & $(.16)$ & $(-.49)$ & $(.37)$ & $(1.30)$ & $(.68)$ & $(.87)$ \\
\hline \multirow{2}{*}{$\begin{array}{l}\text { Recently } \\
\text { moved }\end{array}$} & $.05^{* * *}$ & .02 & .01 & -.03 & $.05^{*}$ & .03 & $.11^{* * *}$ & $.11^{\star \star *}$ \\
\hline & $(.18)$ & $(.13)$ & $(.05)$ & $(-.26)$ & (.18) & $(.16)$ & $(.34)$ & $(.44)$ \\
\hline \multirow[t]{2}{*}{ Religiosity } & $-.11^{* * *}$ & $-.13^{* * *}$ & $-.10^{* * *}$ & $-.13^{* * *}$ & $-.10^{* * *}$ & $-.11^{* * *}$ & $-.10^{* * *}$ & $-.10^{* * *}$ \\
\hline & $(-.34)$ & $(-.52)$ & $(-.40)$ & $(-.59)$ & $(-.37)$ & $(-.62)$ & $(-.32)$ & $(-.41)$ \\
\hline \multirow[t]{2}{*}{ Physical Health } & $-.08^{\star \star \star}$ & $-.04^{\star \star \star}$ & $-.11^{* * *}$ & $-.09^{\star \star *}$ & $-.08^{\star \star *}$ & -.02 & $-.02^{* *}$ & $.03^{* *}$ \\
\hline & $(-.50)$ & $(-.35)$ & $(-.77)$ & $(-.81)$ & $(-.49)$ & $(-.15)$ & $(-.14)$ & $(.21)$ \\
\hline \multirow[t]{2}{*}{ Distress } & $.04^{* * *}$ & $.04^{* *}$ & .03 & $.07^{*}$ & $.06^{\star *}$ & $.05^{*}$ & $.05^{\star \star *}$ & .01 \\
\hline & $(.06)$ & (.08) & $(.06)$ & (.15) & $(.07)$ & (.10) & $(.06)$ & $(.02)$ \\
\hline \multirow[t]{2}{*}{ Depression } & .02 & .00 & -.04 & -.01 & $.04^{*}$ & .03 & $.04^{* * *}$ & .01 \\
\hline & (.20) & $(.06)$ & $(-.55)$ & $(-.19)$ & $(.52)$ & $(.55)$ & $(.45)$ & (.19) \\
\hline \multirow[t]{2}{*}{ Black } & $-.03^{\star *}$ & $-.06^{\star * *}$ & -.04 & -.06 & $-.04^{*}$ & -.03 & $-.03^{\star \star *}$ & $-.04^{\star \star *}$ \\
\hline & $(-.62)$ & $(-1.73)$ & $(-1.01)$ & $(-1.76)$ & $(-.76)$ & $(-.88)$ & $(-.48)$ & $(-.81)$ \\
\hline \multirow[t]{2}{*}{ Latino } & $-.05^{\star \star \star}$ & $-.10^{\star \star \star}$ & -.04 & -.03 & $-.07^{\star \star *}$ & $-.07^{\star *}$ & $-.06^{\star \star *}$ & $-.06^{* * *}$ \\
\hline & $(-.76)$ & $(-2.08)$ & $(-1.04)$ & $(-1.02)$ & $(-1.25)$ & $(-1.89)$ & $(-.86)$ & $(-1.06)$ \\
\hline $\mathrm{F}$ & $38.34^{* * *}$ & $22.17^{* * *}$ & $6.59^{\star * *}$ & $4.69^{* * *}$ & $12.26^{\star * *}$ & $5.23^{* * *}$ & $87.21^{* * *}$ & $73.36^{\star * *}$ \\
\hline R-Square & .08 & .06 & .06 & .05 & .07 & .05 & .10 & .09 \\
\hline $\mathrm{N}$ & 5886 & 4750 & 1329 & 1130 & 2209 & 1387 & 9958 & 10243 \\
\hline
\end{tabular}

Note: Significance levels: ${ }^{* * *} p<.01,{ }^{* *} p<.05,{ }^{*} p<.10$; unstandardized coefficients shown in parentheses.

.13 among males). As expected, higher levels of household income, along with higher levels of educational attainment are also positively associated with alcohol consumption among both sexes (perhaps indicating a broader social class effect). Having children in the home, on the other hand, significantly lowers the rate of drinking among first-married individuals, and particularly so among females. Interestingly, being employed, along with having moved recently, is significantly associated with higher levels of alcohol consumption among females, yet no significant effects are shown for these characteristics in the model for first-married males. In terms of health characteristics, though, both first-married females and males appear to drink less as a function of poor physical health, and drink significantly more when they have experienced psychological distress.

Among remarried individuals, physical health is also shown to be significantly associated with lower levels of alcohol consumption (beta $=-.11$ among females and .09 among males). As compared to first-married individuals, however, psychological distress does not seem as substantial an influence upon the drinking 
behaviors of remarried individuals. Religious service attendance, though, is a salient determinant of alcohol consumption among remarried females and males, in a manner which is quite comparable to that shown among first-married individuals. The presence of children in the home does appear to decrease drinking behaviors among remarried females, but no significant comparable association is shown in the model for remarried males. This may, of course, be reflective of the nature of remarriage and the unique parent-child relationships which occur therein, particularly in regard to stepfather-stepchild relations.
By comparison, divorced males and females are both shown to drink less alcohol when they have higher household incomes. It is interesting to note, though, that divorced females are shown to consume significantly less alcohol when they are obtaining some form of government welfare support (beta $=-.07$ ). Given that women are more likely than men to experience economic distress and poverty, following a divorce, this effect is not entirely surprising. It is worth noting, however, that divorced women are also significantly less likely to drink when their physical health is poor (beta $=-.08$ ). No significant effects are

Table 4: Ordinary Least Squares Regression Models of Cigarette Usage, by Marital Status

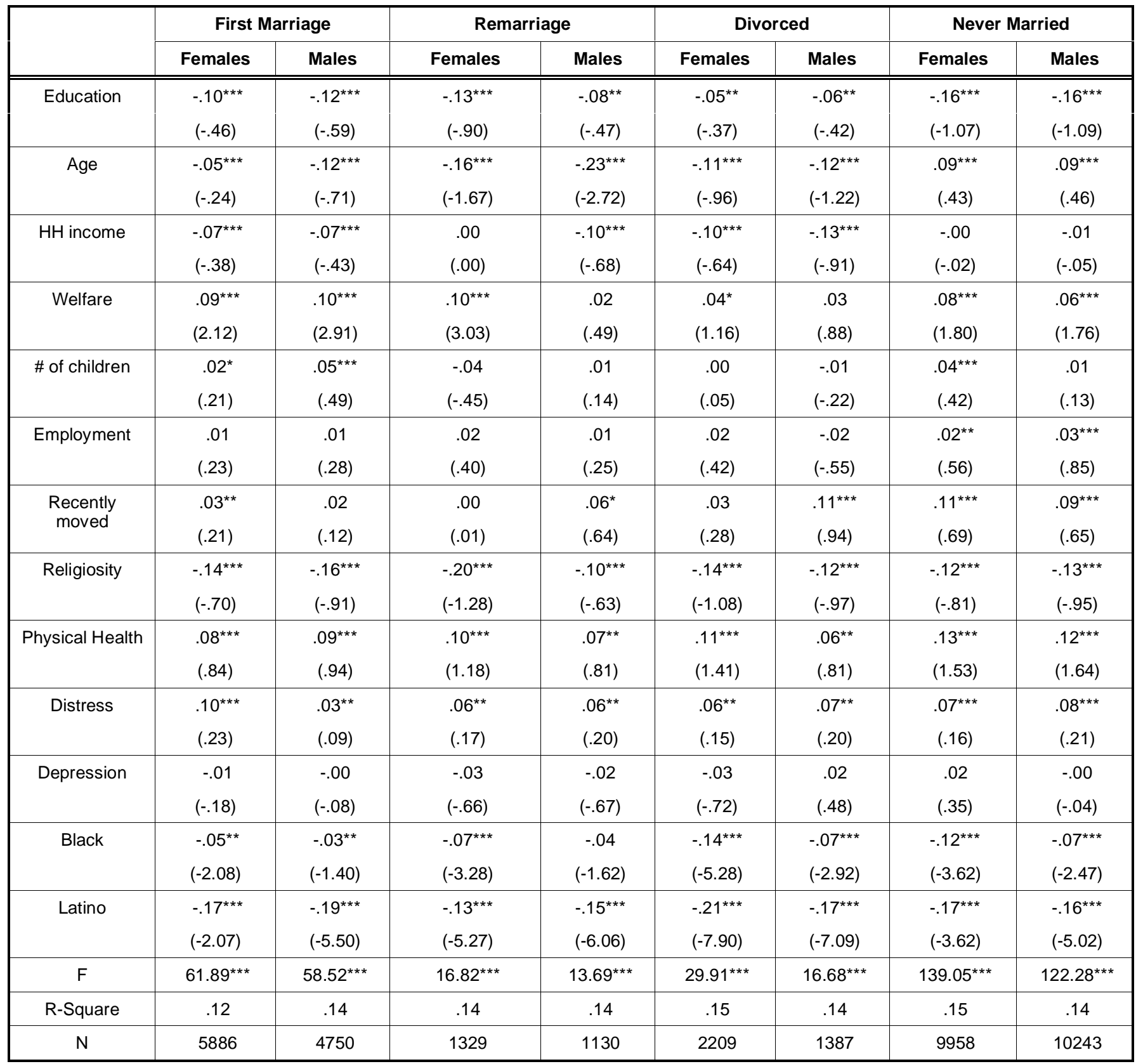

Note: Significance levels: ${ }^{\star \star \star} p<.01,{ }^{\star \star} p<.05,{ }^{\star} p<.10$; unstandardized coefficients shown in parentheses. 
yielded for either welfare support or physical health in the models of divorced men's alcohol usage. Indeed, divorced women are shown to have drink significantly more when they are depressed (beta $=.04$ ), while no comparable association is shown among divorced men. Once more, this may speak to the nature of women's and men's disparate experiences in their post-marital lives.

Among never-married females and males, higher levels of educational attainment are significantly associated with higher rates of drinking. For both sexes, though, the presence of children in the home, along with higher rates of religious service attendance, are significantly associated with lower rates of alcohol consumption. It is interesting to note that religious service attendance yields a relatively consistent influence on drinking behaviors, regardless of sex or marital status. Health-related characteristics, on the other hand, yield quite different effects upon the drinking behaviors of never-married individuals. Among never-married females, poor health is associated with lower rates of alcohol consumption (beta $=-.02$ ), while poor health among never-married males is associated with higher rates of drinking (beta $=.03$ ). Granted, these effects are not among the strongest within their respective models, yet the disparate nature of the associations is certainly worth noting. In conjunction with those differences, never-married females are shown to drink significantly more when they are depressed (beta $=.04$ ) and have experienced higher levels of psychological distress (beta $=.05$ ). Their male counterparts, however, are not shown to be significantly affected by these same qualities.

Table 4 presents the results of the ordinary least squares regression models for cigarette use, by sex and marital status. Among first-married individuals, higher levels of educational attainment are shown to be associated with significantly lower rates of smoking among both sexes. Similarly, higher levels of household income are also associated with lower rates of smoking among first-married females and males. Interestingly, having some form of government welfare support is associated with higher rates of cigarette use among both first-married females and males. Oddly, having children in the home appears to have a more salient positive influence upon the cigarette use by firstmarried males, as compared to their female counterparts. As shown in the models of alcohol consumption, higher levels of religious service attendance are associated with significantly lower rates of cigarette use by both sexes of first-married individuals (beta $=-.14$ among females and -.16 among males). In regard to health, poorer levels of physical health are shown to be linked with higher rates of cigarette use for both sexes of first-married individuals. Of course, smoking, in and of itself, may be related to poor physical health, which makes this association likely to be one of a reciprocal nature. In a similar manner, higher levels of psychological distress are also associated with higher rates of smoking among both first-married females and first-married males.

In the models for cigarette usage by remarried individuals, higher levels of educational attainment are again associated with lower rates of smoking, for both females and males. Interestingly, higher levels of household income appear to significantly lower remarried males' smoking behaviors, yet no significant effect of income is yielded in the model for remarried females. Remarried women are shown to be significantly more likely to smoke when they are receiving some form of government welfare support (beta $=.10)$. As with first-married individuals, remarried females and males appear to smoke significantly less when they attend religious services more often (beta $=$ -.20 among females and -.10 among males). Similarly, physical health is shown to be an important determinant of smoking behaviors, as poorer health is significantly linked with higher rates of smoking. Psychological distress is also significantly associated with higher rates of cigarette use, for both remarried females and remarried males.

Among divorced individuals, higher levels of both educational attainment and household income are significantly associated with lower rates of cigarette use by both females and males. Akin to both first-married and remarried individuals, divorced females and males are shown to be less likely to smoke as they age (beta $=-.11$ among females and -.12 among males). Interestingly, divorced males who have recently moved are shown to smoke cigarettes considerably more often, while moving does not yield a significant effect in the model for divorced females. Religiosity is once more an important determinant of smoking behaviors among divorced individuals, with higher rates of religious service attendance being linked to significantly reduced smoking rates for both sexes. Poor physical health among divorced individuals is significantly associated with higher rates of cigarette use, while psychological distress appears to significantly increase smoking rates for both divorced females and divorced males. 
Both never-married females and males are shown to smoke less, as a function of higher levels of educational attainment. Among never-married individuals, though, age is associated with a higher rate of smoking for both sexes, which is contrary to the effects of age shown among married and previously married individuals. This is likely due to the relatively younger age of the never-married population, and should not necessary be attributed directly to their marital status, per se. It is worth noting that the presence of children is associated with a higher rate of smoking among never-married females (beta $=.04$ ), while this effect is not shown to be significant among never-married males. Having moved recently is significantly associated with higher rates of smoking for both never-married females and males. As shown among individuals of other marital statuses, nevermarried individuals who attend religious services more frequently appear to smoke significantly less, as a function of religiosity. Psychological distress, though, is associated with higher rates of smoking among both never-married females (beta $=.07$ ) and never-married males $($ beta $=.08)$.

Table 5: Ordinary Least Squares Regression Models of Marijuana Usage, by Marital Status

\begin{tabular}{|c|c|c|c|c|c|c|c|c|}
\hline & \multicolumn{2}{|c|}{ First Marriage } & \multicolumn{2}{|c|}{ Remarriage } & \multicolumn{2}{|c|}{ Divorced } & \multicolumn{2}{|c|}{ Never Married } \\
\hline & Females & Males & Females & Males & Females & Males & Females & Males \\
\hline \multirow[t]{2}{*}{ Education } & -.01 & $-.05^{\star * *}$ & -.04 & -.05 & -.00 & -.03 & $-.04^{* * *}$ & -.02 \\
\hline & $(-.01)$ & $(-.12)$ & $(-.07)$ & $(-.11)$ & $(-.01)$ & $(-.09)$ & $(-.16)$ & $(-.08)$ \\
\hline \multirow[t]{2}{*}{ Age } & $-.05^{\star * *}$ & $-.10^{\star \star *}$ & $-.16^{\star * *}$ & $-.07^{*}$ & $-.08^{* * *}$ & $-.12^{\star \star \star}$ & $-.07^{* * *}$ & $-.08^{* * *}$ \\
\hline & $(-.06)$ & $(-.26)$ & $(-.46)$ & $(-.31)$ & $(-.22)$ & $(-.57)$ & $(-.19)$ & $(-.30)$ \\
\hline \multirow[t]{2}{*}{$\mathrm{HH}$ income } & $-.03^{*}$ & $-.04^{\star \star}$ & -.00 & .06 & -.02 & $-.06^{*}$ & $.02^{*}$ & $-.02^{*}$ \\
\hline & $(-.04)$ & $(-.11)$ & $(-.01)$ & $(.15)$ & $(-.04)$ & $(-.19)$ & $(.06)$ & $(-.08)$ \\
\hline \multirow[t]{2}{*}{ Welfare } & .01 & .01 & .01 & .03 & $.04^{*}$ & -.04 & $.04^{* * *}$ & $.05^{\star * *}$ \\
\hline & $(.09)$ & (.12) & $(.10)$ & $(.41)$ & $(.37)$ & $(-.51)$ & $(.50)$ & $(.96)$ \\
\hline \multirow[t]{2}{*}{ \# of children } & -.01 & $.03^{*}$ & $-.12^{\star \star *}$ & .05 & -.00 & -.00 & $-.06^{\star \star *}$ & $-.03^{* * *}$ \\
\hline & $(-.02)$ & $(.12)$ & $(-.39)$ & $(.22)$ & $(-.06)$ & $(-.02)$ & $(-.38)$ & $(-.31)$ \\
\hline \multirow[t]{2}{*}{ Employment } & .00 & $-.03^{*}$ & -.01 & -.06 & -.02 & .01 & $.05^{* * *}$ & .01 \\
\hline & $(.01)$ & $(-.34)$ & $(-.04)$ & $(-.55)$ & $(-.18)$ & (.19) & (.63) & (.23) \\
\hline \multirow{2}{*}{$\begin{array}{l}\text { Recently } \\
\text { moved }\end{array}$} & $.03^{*}$ & -.00 & $.08^{\star \star \star}$ & .01 & .01 & .03 & $.08^{* * *}$ & $.07^{\star \star \star}$ \\
\hline & (.05) & $(-.01)$ & $(.22)$ & $(.03)$ & $(.02)$ & $(.12)$ & $(.28)$ & $(.35)$ \\
\hline \multirow[t]{2}{*}{ Religiosity } & $-.07^{\star \star \star}$ & $-.12^{\star \star \star}$ & -.03 & -.04 & $-.04^{* *}$ & $-.11^{\star * \star}$ & $-.11^{\star * *}$ & $-.12^{* \star *}$ \\
\hline & $(-.09)$ & $(-.30)$ & $(-.05)$ & $(-.10)$ & $(-.11)$ & $(-.41)$ & $(-.38)$ & $(-.63)$ \\
\hline \multirow[t]{2}{*}{ Physical Health } & $.03^{*}$ & .02 & .02 & .04 & -.01 & .00 & $.06^{* * *}$ & $.07^{\star \star \star}$ \\
\hline & $(.08)$ & $(.11)$ & $(.06)$ & (.19) & $(-.04)$ & (.03) & $(.41)$ & $(.66)$ \\
\hline \multirow[t]{2}{*}{ Distress } & $.04^{* * *}$ & $.05^{\star * *}$ & $.08^{\star *}$ & .03 & .03 & $.08^{* * *}$ & $.07^{* * *}$ & $.04^{\star \star *}$ \\
\hline & $(.03)$ & $(.07)$ & $(.06)$ & $(.04)$ & (.03) & $(.11)$ & $(.09)$ & $(.08)$ \\
\hline \multirow[t]{2}{*}{ Depression } & $.03^{* *}$ & $.05^{* * *}$ & .03 & .02 & $.04^{*}$ & -.01 & $.03^{* *}$ & $.03^{* *}$ \\
\hline & $(.16)$ & $(.54)$ & (.19) & (.19) & $(.36)$ & $(-.14)$ & (.33) & $(.48)$ \\
\hline \multirow[t]{2}{*}{ Black } & .02 & $.05^{\star * *}$ & -.01 & -.05 & .03 & -.01 & $.04^{* * *}$ & -.00 \\
\hline & $(.17)$ & $(.87)$ & $(-.06)$ & $(-.78)$ & (.34) & $(-.09)$ & $(.58)$ & $(-.03)$ \\
\hline \multirow[t]{2}{*}{ Latino } & $-.02^{*}$ & $-.08^{\star \star *}$ & $-.05^{*}$ & -.02 & -.01 & $-.09^{\star \star *}$ & $-.02^{\star *}$ & $-.06^{* * *}$ \\
\hline & $(-.17)$ & $(-1.11)$ & $(-.54)$ & $(-.31)$ & $(-.08)$ & $(-1.77)$ & $(-.37)$ & $(-1.16)$ \\
\hline $\mathrm{F}$ & $7.17^{\star \star \star}$ & $19.99^{* * *}$ & $5.84^{* * *}$ & $2.35^{\star * *}$ & $3.66^{\star * *}$ & $5.47^{* \star *}$ & $38.26^{\star * *}$ & $36.21^{* * \star}$ \\
\hline R-Square & .02 & .05 & .06 & .03 & .02 & .05 & .05 & .04 \\
\hline $\mathrm{N}$ & 5886 & 4750 & 1329 & 1130 & 2209 & 1387 & 9958 & 10243 \\
\hline
\end{tabular}

Note: Significance levels: ${ }^{* *} \mathrm{p}<.01,{ }^{* *} \mathrm{p}<.05,{ }^{*} \mathrm{p}<.10$; unstandardized coefficients shown in parentheses. 
Table 5 contains the results of the ordinary least squares regression models for marijuana usage, by sex and marital status. In the models of first-married individuals, age is significantly associated with lower rates of marijuana usage, by both females and males. Household income yields a similar influence for both sexes of first-married individuals. Interestingly, the presence of children in the home significantly increases marijuana usage among males, yet does not significantly affect female usage. Similarly, employment is associated with lower rates of marijuana use among first-married males (beta $=-.03$ ), yet this same association is not significant in the model for firstmarried females. Religious service attendance appears to influence first-married females and males in a similar manner, with higher religiosity shown to be associated with lower rates of marijuana use. Females with poor physical health appear to use marijuana more frequently than their male counterparts. Higher rates of psychological distress and depression, on the other hand, are significantly linked to higher rates of marijuana use by both first-married females and males.

Among remarried individuals, age is negatively associated with marijuana use rates, yet this effect appears to be slightly more salient among remarried females, as compared to males ( $b=-.46$ versus -.31 ). Having children in the home serves to significantly reduce marijuana consumption among remarried females (beta $=-.12$ ), yet children yield no significant effect upon the marijuana use by remarried males. In a contrary manner, having moved recently is associated with higher rates of marijuana use by remarried females, but not their male counterparts. Similarly, higher levels of psychological distress are positively associated with remarried females' marijuana use, but no significant effect is shown for this characteristic in the model for remarried males. It is also worth noting that, as compared to individuals from other marital statuses, remarried females and males are not significantly influenced by religious service attendance, in regard to their use of marijuana.

Both divorced females' and divorced males' use of marijuana is negatively associated with age, such that older individuals appear to use less. Religious service attendance also yields significant influence upon marijuana use among divorced females and males (beta $=-.04$ and -.11 , respectively). Oddly, psychological distress is shown to be positively associated with divorced males' use of marijuana, yet not with females' marijuana usage. Experiencing depression, however, is positively associated with divorced females' use of marijuana, yet not with males' usage. This may again underscore the rather distinct experiences of women and men, as they deal with divorce and its eventual consequences.

Finally, among never-married individuals, age also yields a negative association with marijuana usage by both sexes. Interestingly, the receipt of government welfare support is positively associated with marijuana usage by both never-married females and nevermarried males. Having children in the home, though, yields the opposite effect, and is linked with lower rates of marijuana usage by never-married individuals. It is rather noteworthy that employment is associated with significantly higher rates of marijuana use by nevermarried females, yet paid work does not yield a significant effect in the model for never-married males. Having recently moved appears to affect never-married females and males in a similar manner, such that it is associated with higher rates of marijuana use. Religious service attendance is also negatively associated with marijuana use among never-married individuals of both sexes. Lastly, the measures of health and well-being yield fairly consistent effects for both sexes, in terms of marijuana use among nevermarried individuals. Poor physical health is associated with higher rates of marijuana use, as are higher levels of psychological distress and depression, among both never-married females and never-married males.

\section{CONCLUSIONS AND DISCUSSION}

This study was initiated with the purpose of examining differences in substance use across marital statuses, as well as to better understand the differences between females' and males' usage patterns, therein. Previous studies had noted changes in substance use levels, specifically as individuals transitioned from single to married status, yet no study had made a direct comparison of substance use levels across all possible marital statuses. The results from these analyses clearly support the contention that marital status does, in fact, have a substantial bearing upon substance use levels by individuals in the U. S. Additionally, the gender differences shown suggest that the experiences within each marital status have unique effects upon each sex, and these vary, depending upon the substance in questions. Clearly, these patterns will necessitate further scrutiny.

Given the sometimes turbulent and disruptive nature of divorce, it was assumed that divorced individuals would report the highest levels of substance 
use, across all three types, alcohol, cigarettes, and marijuana. However, this was not found to be the case. While divorced women and men did report the highest rates of smoking, as compared to those of other marital statuses, the highest rates of marijuana usage were reported among the never-married respondents. In regard to alcohol, divorced men reported the highest rates of drinking among the males, while among women, it was remarried females who had the heaviest rates of alcohol consumption.

Individuals in their first marriage, both female and male alike, reported the lowest rates of substance use, as compared to the other marital statuses. This is in keeping with previous studies which had noted a decline among individuals as they transitioned into marriage. The sample of first-married individuals in this study, though, also contains individuals who are well into their adult lives, and have been married, in some instances, for several decades. As such, it is also necessary to recognize that the transitional nature of first marriage does not appear to be a limited or timeconstrained effect. Rather, on the basis of these findings, individuals in their first marriage clearly seem to have made a conscious choice to use lower amounts of the various substances, if at all. The decidedly high rates of non-use among first-married individuals seems to support this contention.

The various household and individual characteristics yielded several rather intriguing patterns within the multivariate analyses. Among first-married individuals, household income was shown to significantly influence their use of all three substances. Among the other marital status groups, though, household income was not as relevant. Of course, for many first-married individuals, and particularly those in their early adult years, concerns about family finances may lower their ability to purchase various substances, legal or otherwise. Surprisingly, the presence of children did not yield as significant an influence, as anticipated. Although remarried women were shown to use less marijuana when children were present in the home, parenting (assumed with the presence of children) does not seem to equate with a direct reduction or rejection of substance use.

The unique influence of religiosity on substance use, as was evident across all marital status groups, is also quite noteworthy. Previous studies have demonstrated that higher levels of religiosity are significantly associated with lower rates of substance use (e.g., Bahr et al., 1998). The fact that this relationship persists across marital status groups lends even further weight to religiosity as a reliable predictor of substance use rates. Well-being, both in the physical and mental regards, was also shown to be a salient factor in affecting substance use rates among women and men, across all marital statuses. Overall, poor mental well-being appears to be associated with higher rates of usage, among all three of the substances, while poor physical health has the opposite association. Physical health seemed to have its strongest effect upon the substance use patterns among remarried and divorced women, which again suggests a 'gendered divide' in how each respective sex experiences the various marital statuses, as well as the associated consequences thereof.

Of course, it is necessary to recognize the potential for reciprocal influences in the relationship between marital status and substance use. Researchers have demonstrated that higher levels of substance use by a partner can be deleterious to the quality of the relationship, and can potentially lead to its dissolution (e.g., Kearns-Bodkin and Leonard, 2005). Likewise, it is quite conceivable that substance use patterns by individuals can contribute to the mate selection process itself, thereby leading some to choose partners who share their preferences for substance use (e.g., Leonard and Mudar, 2003). These findings, however, seem to clearly demonstrate that marital status, in and of itself, is a solid predictor of substance use, and that each marital status provides a unique context in which those usage patterns occur. The differences between women's and men's experiences also appear to confirm the long-standing belief that each sex, regardless of their marital status, has their own unique set of both contexts and consequences. Obviously, these findings are limited in their scope, largely to the nature of the data, but they do demonstrate the need for further investigation. In this regard, future research should attempt to more precisely focus on the unique qualities of each marital status, as they potentially affect substance use rates by women and men.

\section{REFERENCES}

Bachman, J. G.; O’Malley, P. M.; Schulenberg, J. E.; Johnston, L. D.; Bryant, A. L.; and Merline, A.C. (2002). The decline in substance use in young adulthood: Changes in social activities, roles, and beliefs. Mahwah, N. J.: Lawrence Erlbaum Associates.

Bachman, J. G.; Wadsworth, K. N.; O'Malley, P. M.; Johnston, L. D.; and J. E. Schulenberg. (1997). Smoking, drinking, and drug use in young adulthood: The impacts of new freedoms and new responsibilities. Mahwah, N. J.: Lawrence Erlbaum Associates. 
Bahr, S. J.; Maughan, S. L.; Marcos, A. C.; and B. Li. (1998). Family, religiosity, and the risk of adolescent drug use. Journal of Marriage and the Family, 60, 979-992. http://dx.doi.org/10.2307/353639

Blair, S. L. (2010). The influence of risk-taking behaviors on the transition into marriage: An examination of the long-term consequences of adolescent behavior. Marriage and Family Review, 46, 126-146. http://dx.doi.org/10.1080/01494921003685169

Chen, K. and Kandel, D. B. (1995). Predictors of cessation of marijuana use: An event history analysis. Drug and Alcohol Dependence, 50, 2, 109-121. http://dx.doi.org/10.1016/S0376-8716(98)00021-0

Ehrenberg, M. F.; Robertson, M.; and Pringle, J. (2012). Attachment style and marital commitment in the context of remarriage. Journal of Divorce and Remarriage, 53, 204- 219. http://dx.doi.org/10.1080/10502556.2012.663270

Fischer, T. F. C.; De Graaf, P. M.; and Kalmijn, M. (2005). Friendly and antagonistic contact between former spouses after divorce. Journal of Family Issues, 26, 8, 1131-1163. http://dx.doi.org/10.1177/0192513X05275435

Fleming, C. B.; White, H. R.; and R. F. Catalano. (2010). Romantic relationships and substance use in early adulthood: An examination of the influences of relationship type, partner substance use, and relationship quality. Journal of Health and Social Behavior, 51, 2, 153-167. http://dx.doi.org/10.1177/0022146510368930

Furukawa, T. A., Kessler, R. C., Slade, T., \& Andrews, G. (2003). The performance of the $\mathrm{K} 6$ and $\mathrm{K} 10$ screening scales for psychological distress in the Australian National Survey of Mental Health and Well-Being. Psychological Medicine, 33(2), 357-362.

http://dx.doi.org/10.1017/S0033291702006700

Giordano, P. C.; Cernkovich, S. A.; and J. L. Rudolph. (2002). Gender, crime, and desistance: Toward a theory of cognitive transformation. American Journal of Sociology, 107, 9901064.

http://dx.doi.org/10.1086/343191

Graber, J. A. and J. Brooks-Gunn. (1996). Transitions and turning points: Navigating the passage from childhood through adolescence. Developmental Psychology, 32, 4, 768-776. http://dx.doi.org/10.1037/0012-1649.32.4.768

Horney, J.; Osgood, D. W.; and I. H. Marshall. (1995). Criminal careers in the short term: Intra- individual variability in crime and its relation to local life circumstances. American Sociological Review, 60, 655-673. http://dx.doi.org/10.2307/2096316

Horwitz, A. V.; White, H. R.; and S. Howell-White. (1996). Becoming married and mental health: A longitudinal study of a cohort of young adults. Journal of Marriage and the Family, 4, 895907.

http://dx.doi.org/10.2307/353978

Kearns-Bodkin, J. N. and K. E. Leonard. (2005). Alcohol involvement and marital quality in the early years of marriage: $A$ longitudinal growth curve analysis. Alcoholism: Clinical and Experimental Research, 29, 2123-2134.

http://dx.doi.org/10.1097/01.alc.0000191751.62025.77

Kessler, R. C., Barker, P. R., Colpe, L. J., Epstein, J. F., Groerer, J. C., Hiripi, E., Howes, M. J., Normand, S. L., Manderscheid, R. W., Walters, E. E., \& Zaslavsky, A. M. (2003). Screening for serious mental illness in the general population. Archives of General Psychiatry, 60(2), 184-189.

http://dx.doi.org/10.1001/archpsyc.60.2.184

Kreider, R. M. and Fields, J. M. (2002). Number, timing, and duration of marriages and divorces: 1996. Current Population Reports, P70-80. Washington, D.C.: U.S. Census Bureau.

Leonard, K. E. and Homish, G. G. (2005). Changes in marijuana use over the transition into marriage. Journal of Drug Issues, 35 , $2,409-429$ http://dx.doi.org/10.1177/002204260503500209

Leonard, K. E. and P. Mudar. (2003). Peer and partner drinking and the transition to marriage: A longitudinal examination of selection and influence processes. Psychology of Addictive Behaviors, 17, 115-125. http://dx.doi.org/10.1037/0893-164X.17.2.115

Maume, M. O.; Ousey, G. C.; and K. Beaver. (2005). Cutting the grass: A reexamination of the link between marital attachment, delinquent peers, and desistance from marijuana use. Journal of Quantitative Criminology, 21, 27-53. http://dx.doi.org/10.1007/s10940-004-1786-3

McGoldrick, M. and Cater, E. A. (1982). The Family Life Cycle. In F. Walsh (Ed.), Normal family processes (pp. 167-195). New York: Guilford Press.

Merline, A. C.; Schulenberg, J. E.; O'Malley, P. M.; Bachman, J. G.; and L. D. Johnston. (2008) Substance use in marital dyads: Premarital assortment and change over time. Journal of Studies on Alcohol and Drugs, May, 352-361. http://dx.doi.org/10.15288/jsad.2008.69.352

Mirecki, R. M.; Chou, J. L.; Elliott, M.; and C. M. Schneider. (2013). What factors influence marital satisfaction? Differences between first and second marriages. Journal of Divorce and Remarriage, 54, 78-93. http://dx.doi.org/10.1080/10502556.2012.743831

Muthen, B. O. and Muthen, L. K. (2000). The development of heavy drinking and alcohol- related problems from ages 18 to 37 in a U.S. national sample. Journal of Studies on Alcohol, 61, 2 290-300. http://dx.doi.org/10.15288/jsa.2000.61.290

Nock, S. L. (1998). The consequences of premarital fatherhood. American Sociological Review, 63, 250-263. http://dx.doi.org/10.2307/2657326

Saint-Jacques, M. C.; Rabitaille, C.; Godbout, E.; Parent, C.; Drapeau, S.; and M. H. Gagne. (2011). The processes distinguishing stable from unstable stepfamily couples: A qualitative analysis. Family Relations, 60, 545-561. http://dx.doi.org/10.1111/i.1741-3729.2011.00668.x

Substance Abuse and Mental Health Services Administration (Office of Applied Studies). (2003). Results from the 2002 National Survey on Drug Use and Health: National Findings, DHHS Publication No. SMA 03-3836. Rockville, Maryland: Substance Abuse and Mental Health Services Administration.

Sweeney, M. M. (1997). Remarriage of women and men after divorce: The role of socioeconomic prospects. Journal of Family Issues, 18, 479-502. http://dx.doi.org/10.1177/019251397018005002

U.S. Census Bureau. (2012). Statistical Abstract of the United States: 2012 (131st Edition) Washington, D.C.: U.S. Government Printing Office.

\section{DOI: http://dx.doi.org/10.6000/1929-4409.2016.05.01}

(c) 2016 Blair and Menasco; Licensee Lifescience Global.

This is an open access article licensed under the terms of the Creative Commons Attribution Non-Commercial License (http://creativecommons.org/licenses/by-nc/3.0/) which permits unrestricted, non-commercial use, distribution and reproduction in any medium, provided the work is properly cited. 